$$
\begin{array}{r}
\text { Pontifícia Universidade C Católica } \\
\text { do Rio de Janeiro }
\end{array}
$$

Janaina Cunha e Silva Arteaga

\title{
Aplicação de Método de Elementos Finitos na Análise de Variações de Campo em Estruturas Coaxiais devido a Perturbações nas Condições de Contorno
}

\section{Dissertação de Mestrado}

Dissertação apresentada como requisito parcial para obtenção do grau de Mestre pelo Programa de Pósgraduação em Engenharia Elétrica do Departamento de Engenharia Elétrica da PUC-Rio.

Orientador: Prof. José Ricardo Bergmann

Rio de Janeiro 


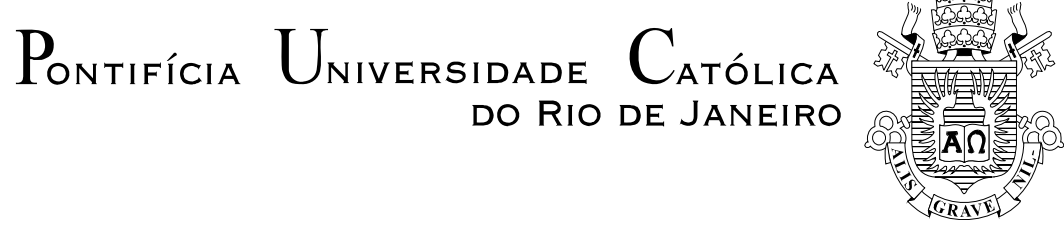

Janaina Cunha e Silva Arteaga

\begin{abstract}
Aplicação de Método de Elementos Finitos na Análise de Variações de Campo em Estruturas Coaxiais devido a Perturbações nas Condições de Contorno
\end{abstract}

Dissertação apresentada como requisito parcial para obtenção do título de Mestre pelo Programa de PósGraduação em Engenharia Elétrica do Departamento de Engenharia Elétrica do Centro Técnico Científico da PUCRio. Aprovada pela Comissão Examinadora abaixo assinada.

\title{
Dr. José Ricardo Bergmann
}

Orientador

Centro de Estudos em Telecomunicações/PUC-Rio

Dr. Flávio José Vieira Hasselmann

Centro de Estudos em Telecomunicações/PUC-Rio

Dr. Luiz Costa da Silva

Centro de Estudos em Telecomunicações/PUC-Rio

Dr. Fernando José da Silva Moreira

UFMG

José Eugênio Leal

Coordenador Setorial do Centro

Técnico Científico - PUC-Rio

Rio de Janeiro, 05 de novembro de 2007 
Todos os direitos reservados. É proibida a reprodução total ou parcial do trabalho sem autorização da universidade, do autor e do orientador.

\section{Janaina Cunha e Silva Arteaga}

Graduou-se em Engenharia Elétrica, em dezembro de 2004, na Universidade Federal de Minas Gerais. Em março de 2005, iniciou no Centro de Estudos em Telecomunicações da Pontifícia Universidade Católica do Rio de Janeiro seu mestrado na área de eletromagnetismo aplicado.

Ficha Catalográfica

Arteaga, Janaina Cunha e Silva

Aplicação de método de elementos finitos na análise de variações de campo em estruturas coaxiais devido a perturbações nas condições de contorno / Janaina Cunha e Silva Arteaga ; orientador: José Ricardo Bergmann. - 2007.

102 f. ; $30 \mathrm{~cm}$

Dissertação (Mestrado em Engenharia Elétrica) Pontifícia Universidade Católica do Rio de Janeiro, Rio de Janeiro, 2007.

Inclui bibliografia

1. Engenharia elétrica - Teses. 2. Método de elementos finitos. 3. Guias coaxiais. 4. Perturbações em dutos. I. Bergmann, José Ricardo. II. Pontifícia Universidade Católica do Rio de Janeiro. Departamento de Engenharia Elétrica. III. Título. 


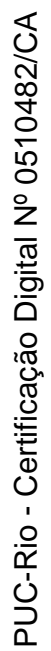

Á minha Família. 


\section{Agradecimentos}

Ao meu orientador Professor José Ricardo Bergmann pelo estímulo, apoio e parceria no decorrer desta dissertação.

Ao CNPq, a FAPERJ, à PUC-Rio e à PipeWay, pelos auxílios concedidos, sem os quais esta dissertação não poderia ter sido realizada.

À minha família, pela educação, atenção e carinho de todas as horas.

A todos os amigos do CETUC que de alguma forma me estimularam ou ajudaram. 


\section{Resumo}

Arteaga, Janaina Cunha e Silva; Bergmann, José Ricardo (Orientador). Aplicação de Método de Elementos Finitos na Análise de Variações de Campo em Estruturas Coaxiais devido a Perturbações nas Condições de Contorno. Rio de Janeiro, 2007. 102p. Dissertação de Mestrado Departamento de Engenharia Elétrica, Pontifícia Universidade Católica do Rio de Janeiro.

Neste trabalho, o Método dos Elementos Finitos (MEF) é aplicado na análise do campo eletromagnético em dutos metálicos, utilizados no transporte de óleo e gás. Instrumentos de inspeção podem utilizar esse método para verificar a existência de anomalias do tipo amassamentos e corrosões nos dutos, contribuindo para evitar vazamentos que podem causar acidentes ecológicos. Atualmente, a técnica de fuga de campo magnético (MFL) é a mais utilizada para precisar a profundidade dos defeitos encontrados nos dutos devido ao processo localizado de corrosão, onde a espessura se reduz progressivamente até o inicio de um eventual vazamento. Os picos dos sinais de fluxo magnético defletido indicam a perda de massa metálica localizada. A profundidade dos defeitos é correlacionada com a amplitude dos picos. Neste trabalho, o algoritmo baseado em Método de Elementos Finitos é utilizado para avaliar o desempenho do MEF na localização da posição de defeitos na parede do duto, através dos picos que aparecem nos gráficos de campo magnético. Essa análise será feita utilizando-se dois tipos de alimentação na entrada do duto: (I) apenas propagação do modo fundamental TEM e (II) diferença de potencial entre os cilindros externo e interno do duto. Diversos tamanhos de deformação em posições diferentes do duto serão analisados para se determinar para quais tipos de problema o algoritmo é eficiente.

\section{Palavras-chave}

Método de Elementos Finitos, Guias Coaxiais, Perturbações em dutos. 


\section{Abstract}

Arteaga, Janaina Cunha e Silva; Bergmann, José Ricardo (Advisor). Application of Finite Element Method in the Analysis of Filed Variations in Coaxial Structures due to Perturbation in the Boundary Conditions. Rio de Janeiro, 2007. 102p. MSc Dissertation - Departamento de Engenharia Elétrica, Pontifícia Universidade Católica do Rio de Janeiro.

In this work, the Finite Element Method (FEM) is applied in the analysis of electromagnetic field generated by instruments employed for inspection of pipelines for natural gas-transmission. Inspection instruments can use this method to evaluate anomalies as corrosion and kneading in the superficies of the pipelines. This can avoid leaking that can cause ecological accidents. The Magnetic Flux Leakage (MFL) is the oldest and most commonly used in-line inspection method for finding metal-loss regions in gas-transmission pipelines. MFL inspections are typically used to detect, locate, and characterize metal-loss and other anomalies in natural gas-transmission pipelines. The amplitude or magnitude of an MFL signal is strongly related to defect depth.. Alternatively, in this work, an instrument that generates a TEM wave is explored for inspection of pipelines. To evaluate the field distribution inside the pipe, an algorithm based on Finite Element Method is used to detect and locate an anomaly in the pipeline. It is used two kinds of source in the duct's port: (I) just propagation in the FEM basic mode and (II) different potential between the internal and external cylindrical duct. Several sizes of anomalies will be analyzed to identify for which kind of problems the algorithm is useful.

\section{Keywords}

Finite Element Methods, Coaxial wave guides, Pipelines. 


\section{Sumário}

1 Introdução

2 Formulação das Equações

2.1. Introdução

2.2. Formulação Eletromagnética 18

2.3. Equação de Onda

2.4. Excitação do Dispositivo 23

2.5. Método de Galerking 26

2.6. Solução da Equação de Onda Homogênea 29

2.6.1. Tranverse Eletromagnetic Mode (TEM) 311

2.6.2. Transverse Magnetic Mode (TM) 33

3 Método de Elementos Finitos (MEF) 38

3.1. Discretização do Domínio em Elementos 39

3.2. Funções de Base e de Teste (Peso) 39

3.3. Equação do Método de Elementos Finitos em 2D 41

3.4. Polinômio Interpolador $\quad 47$

3.5. Mapeamento e Degeneração 50

3.6. Preenchimento da Matriz 55

3.6.1. Cálculo dos termos $\operatorname{com} \boldsymbol{i}=\boldsymbol{j}$

3.6.2. Cálculo dos termos com $\boldsymbol{i} \neq \boldsymbol{j}$

3.7. Formação da Matriz Global 60

4 Estudo da estrutura cilíndrica 63

4.1. Introdução 63

4.2. Validação do Algoritmo 65

4.3. Estrutura Cilíndrica Com Alimentação Tipo I e Sem Deformações na $\begin{array}{ll}\text { Superfície } & 67\end{array}$

4.4. Estrutura Cilíndrica com Alimentação Tipo I e Com Deformação na Superfície 
4.4.1. Variação da Freqüência de Excitação do Duto 73

4.4.2. Variação da Posição da Ranhura 76

4.4.3. Variação do Tamanho da Deformação 77

4.4.4. Conclusões 81

4.5. Estrutura Cilíndrica Com Alimentação Tipo II e Sem Deformações na Superfície 82

4.6. Estrutura Cilíndrica Com Alimentação Tipo II e Com Deformação na Superfície 85

4.6.1. Variação da Freqüência de Excitação do Duto 90

4.6.2. Variação da Posição da Ranhura 92

4.6.3. Variação do Tamanho da Deformação 94

4.6.4. Conclusões 97

5 Conclusões $\quad 99$

6 Referências bibliográficas 101 


\section{Lista de Figuras}

Figura 1.1.: Dispositivo de inspeção do duto. ...................................................... 16

Figura 2.1.: Geometria do problema proposto. (a) seção de guia de onda coaxial e

(b) sistema de coordenadas cilíndricas [11]. 18

Figura 2.2.: Alimentação Tipo I - modo TEM incidente na porta de entrada....... 19

Figura 2.3.: Alimentação Tipo II - fonte de corrente magnética sobre a porta de

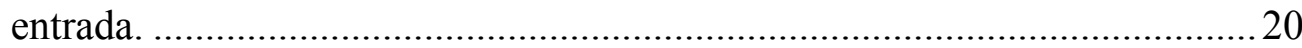

Figura 2.4.: Regiões da seção reta da estrutura cilíndrica. ................................... 21

Figura 2.5.: Propriedades dos meios $1\left(\varepsilon_{1}, \mu_{1}\right)$ no interior do duto e $2\left(\varepsilon_{2}, \mu_{2}\right)$ no

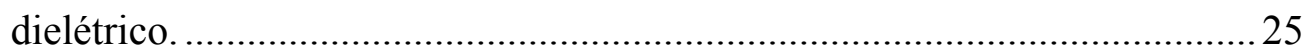

Figura 2.6.: Sistema de coordenadas cilíndricas e vetores unitários

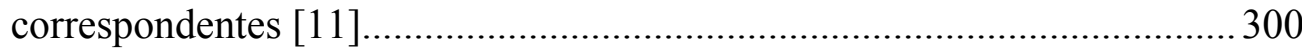

Figura 3.1.: Exemplos de elementos em duas dimensões [4] ...............................39

Figura 3.2.: Funções de subdomínio..................................................................... 40

Figura 3.3.: Corrente magnética na parede 1 (entrada) do guia coaxial em 2D... 43

Figura 3.4.: Triângulo de Pascal e polinômios associados de $\mathrm{N}=0$ até $\mathrm{N}=2$ [7].... 47

Figura 3.5.: Coordenadas dos nós do elemento de ordem 1................................50

Figura 3.6.: Mapeamento de um elemento de primeira ordem.............................50

Figura 3.7.: Função forma para elemento triangular. ...........................................5 52

Figura 3.8.: Sistema de primeira ordem com 4 elementos e 6 nós........................ 61

Figura 4.1.: Estrutura cilíndrica com diferença de potencial V...........................65

Figura 4.2.: Estrutura cilíndrica com alimentação Tipo I e sem dielétrico. ..........65 65

Figura 4.3.: Campo magnético para uma estrutura lisa e sem perdas. .................. 66

Figura 4.4.: Estrutura cilíndrica com alimentação Tipo I...................................... 67

Figura 4.5.: Campo Magnético de um duto sem deformações na superfície,

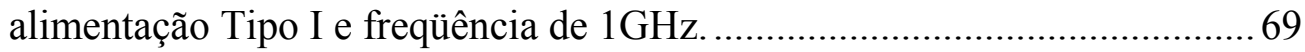

Figura 4.6.: Fase de um duto sem deformações na superfície, alimentação Tipo I e

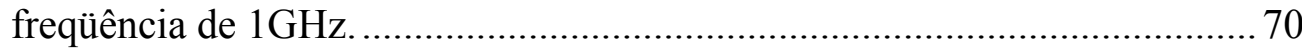

Figura 4.7.: Campo Magnético de um duto sem deformações na superfície e alimentação Tipo I para freqüência de $2 \mathrm{GHz}$.............................................. 71 
Figura 4.8.: Fase de um duto sem deformações na superfície e alimentação Tipo I

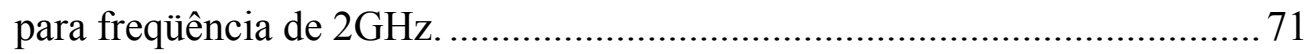

Figura 4.9.: Estrutura cilíndrica com alimentação Tipo I e deformação na superfície. .72

Figura 4.10.: Reflexão de ondas na estrutura com deformação na superfície....... 74

Figura 4.11.: Diferença percentual entre os módulos do campo magnético com e sem deformação. Corrugação entre $\mathrm{z}=5 \mathrm{~cm}$ e $\mathrm{z}=6 \mathrm{~cm}$, com dimensão de $1 \mathrm{cmx} 1 \mathrm{~cm}$. 75

Figura 4.12.: Diferença percentual entre os módulos do campo magnético com e sem deformação. Corrugação entre $\mathrm{z}=10 \mathrm{~cm}$ e $\mathrm{z}=11 \mathrm{~cm}$, com dimensão de $1 \mathrm{cmx} 1 \mathrm{~cm}$ 76

Figura 4.13.: Diferença percentual entre os módulos do campo magnético com e sem deformação. Corrugação entre $\mathrm{z}=5 \mathrm{~cm} \mathrm{e} \mathrm{z}=6 \mathrm{~cm}$, para freqüência $0,5 \mathrm{GHz}$ 78

Figura 4.14.: Diferença percentual entre os módulos do campo magnético com e sem deformação. Corrugação entre $\mathrm{z}=5 \mathrm{~cm}$ e $\mathrm{z}=6 \mathrm{~cm}$, para freqüência 1,0 $\mathrm{GHz}$ .79

Figura 4.15.: Diferença percentual entre os módulos do campo magnético com e sem deformação. Corrugação entre $\mathrm{z}=5 \mathrm{~cm}$ e $\mathrm{z}=6 \mathrm{~cm}$, para freqüência $2,0 \mathrm{GHz}$ 80

Figura 4.16.: Diferença percentual entre os módulos do campo magnético com e sem deformação. Corrugação entre $\mathrm{z}=5 \mathrm{~cm}$ e $\mathrm{z}=6 \mathrm{~cm}$, para freqüência de 2,0 $\mathrm{GHz}$ e dimensão 2,0 x $1,0 \mathrm{~cm}$ 81

Figura 4.17.: Estrutura cilíndrica com alimentação Tipo II. .83

Figura 4.18.: Campo Magnético de um duto sem deformações na superfície e alimentação Tipo II para freqüência de $1 \mathrm{GHz}$............................................ 84

Figura 4.19 - Estrutura cilíndrica com alimentação Tipo II. …………….............. 85

Figura 4.20.: Campo Magnético em um duto com alimentação Tipo II e com deformação na superfície para freqüência de $1 \mathrm{GHz}$ 86

Figura 4.21.: Campo Magnético em um duto com alimentação Tipo II e com deformação na superfície para freqüência de $1 \mathrm{GHz}$ 87

Figura 4.22.: Campo Magnético multiplicado por $\rho$ ao longo da linha constante de $\mathrm{z}=0 \mathrm{~cm}$ para corrugação entre $\mathrm{z}=5$ e $6 \mathrm{~cm}$ e freqüência de $1 \mathrm{GHz}$. 88 
Figura 4.23.: Campo Magnético multiplicado por $\rho$ ao longo de linhas bem próximas de $\mathrm{z}=5 \mathrm{~cm}$ para corrugação entre $\mathrm{z}=5$ e $6 \mathrm{~cm}$ e freqüência de $1 \mathrm{GHz}$. .88

Figura 4.24.: Campo Magnético multiplicado por $\rho$ ao longo de linhas bem próximas de $\mathrm{z}=6 \mathrm{~cm}$ para corrugação entre $\mathrm{z}=5$ e $6 \mathrm{~cm}$ e freqüência de $1 \mathrm{GHz}$.

Figura 4.25.: Campo Magnético multiplicado por $\rho$ ao longo de linhas bem próximas de $\mathrm{z}=21 \mathrm{~cm}$ para corrugação entre $\mathrm{z}=5$ e $6 \mathrm{~cm}$ e freqüência de $1 \mathrm{GHz}$.

Figura 4.26.: Campo Magnético multiplicado por $\rho$ ao longo de linhas bem próximas de $\mathrm{z}=23 \mathrm{~cm}$ para corrugação entre $\mathrm{z}=5$ e $6 \mathrm{~cm}$ e freqüência de $1 \mathrm{GHz}$.

90

Figura 4.27.: Diferença percentual entre os módulos do campo magnético com e sem deformação. Corrugação entre $\mathrm{z}=5 \mathrm{~cm}$ e $\mathrm{z}=6 \mathrm{~cm}$, com profundidade de $1 \mathrm{~cm}$. 91

Figura 4.28.: Diferença percentual entre os módulos do campo magnético com e sem deformação. Corrugação entre $\mathrm{z}=5 \mathrm{~cm}$ e $\mathrm{z}=6 \mathrm{~cm}$, com dimensão de $1 \mathrm{~cm} x 1 \mathrm{~cm}$ e freqüência de $2 \mathrm{GHz}$. 93

Figura 4.29.: Pico Diferença percentual entre os módulos do campo magnético com e sem deformação. Corrugação entre $z=10 \mathrm{~cm}$ e $\mathrm{z}=11 \mathrm{~cm}$, com dimensão de $1 \mathrm{~cm} x 1 \mathrm{~cm}$ e freqüência de $2 \mathrm{GHz}$. 93

Figura 4.30.: Diferença percentual entre os módulos do campo magnético com e sem deformação. Corrugação entre $\mathrm{z}=5 \mathrm{~cm}$ e $\mathrm{z}=6 \mathrm{~cm}$, para freqüência $0,5 \mathrm{GHz}$ 95

Figura 4.31.: Diferença percentual entre os módulos do campo magnético com e sem deformação. Corrugação entre $\mathrm{z}=5 \mathrm{~cm}$ e $\mathrm{z}=6 \mathrm{~cm}$, para freqüência $1 \mathrm{GHz}$. 96

Figura 4.32.: Diferença percentual entre os módulos do campo magnético com e sem deformação. Corrugação entre $\mathrm{z}=5 \mathrm{~cm}$ e $\mathrm{z}=6 \mathrm{~cm}$, para freqüência $2 \mathrm{GHz}$. 


\section{Lista de tabelas}

Tabela 4.1.: Amplitudes das ondas incidentes e refletidas. 69

Tabela 4.2.: Valores dos picos para duto com alimentação Tipo I. 82

Tabela 4.3.: Amplitudes das ondas incidentes e refletidas. 84

Tabela 4.4.: Valores dos picos para duto com alimentação Tipo II. 98 